\title{
Review: family and parenting interventions reduce subsequent arrests and length of time in institutions in youths with conduct disorder and delinquency
}

Woolfenden SR, William K, Peat J. Family and parenting interventions in children and adolescents with conduct disorder and delinquency aged 10-17. Cochrane Database Syst Rev 2001;(2):CD003015 (latest version 28 February 2001).

QUESTION: In youths aged 10-17 years with conduct disorder or delinquency, do family and parenting interventions improve behaviour and long term psychosocial outcomes as well as parenting, family functioning, and parental mental health?

\section{Data sources}

Studies were identified by searching Medline, EMBASE/ Excerpta Medica, PsycINFO, CINAHL, Sociofile, ERIC, and Healthstar (all to September 1999), and by searching the Cochrane Controlled Trials Register. Bibliographies of relevant studies were reviewed, and experts were contacted for informaton on unpublished trials.

Sources of funding: no external funding.

For correspondence: Dr S R Wolfenden, Department of

Psychological Medicine, Children's Hospital at Westmead, Locked Bag 4001, Westmead, New South Wales 2145 Australia.Email susan@chw.edu.au

\section{Data extraction}

2 reviewers independently extracted data on the location of the study, patient characteristics, key components and duration of the intervention, quality of the study, and outcomes.

\section{Main results}

8 studies (749 children and adolescents and their families) were included in the meta-analysis. Family and parenting interventions included short term family intervention, parent training, multisystemic therapy, multidimensional treatment foster care, and an adolescent diversion project with a family condition and a multifocus condition. At follow up, fewer youths in the intervention than in the control group were subsequently arrested (table). Youths in the intervention group spent less time in institutions and had lower self reported delinquency than those in the control group (table). The groups did not differ for youths' behaviour, parental mental health, family functioning and relationships, risk of incarceration, and peer relationships.

\section{Conclusion}

In youths aged 10-17 years with conduct disorder or delinquency, family and parenting interventions reduce the risk of subsequent arrest, length of time spent in institutions, and self reported delinquency compared with usual care or no intervention.

\section{COMMENTARY}

Several studies have examined the efficacy of interventions for conduct disorder and delinquency in children and adolescents. ${ }^{1}$ This systematic review by Woolfenden $e t a l$ is timely as it is the first quantitative synthesis of preventive interventions targeted at youths aged 10-17 years.

The review methodology was rigorous, a thorough search of biomedical and psychosocial bibliographic databases was done, inclusion criteria were specified, and conduct disorder and delinquency were clearly defined. Furthermore, variation within and between studies (heterogeneity) was quantitatively assessed. Although the cost effectiveness of different family and parenting interventions was not evaluated, multisystemic therapy was highlighted as a resource intensive intervention. Only 1 study from Australia targeted conduct disorder; the remaining studies were from the US and included juvenile delinquents who were chronic or serious offenders. The findings, therefore, are based on this population and focus on the US juvenile justice system and thus may not be generalisable outside this setting.

Nevertheless, the results of this systematic review are relevant to nurses working in child and adolescent mental health services and to practitioners whose clients include delinquent juveniles. Recurrent delinquency is associated with high rates of multiple service utilisation and substantial societal cost. Interventions that could reduce the risk of subsequent arrest and time spent in institutions are a valuable contribution to both the adolescent, their family, and society as a whole. Further research is needed on the effectiveness of interventions in children with conduct disorders, particularly given the suggested relation between early conduct problems and risk of subsequent recurrent delinquency. ${ }^{2}$

Julie Bruce, RGN, MSc Research Fellow Dugald Baird Centre for Research on Women's Health, Department of Obstetrics and Gynaecology, University of Aberdeen, Aberdeen, $U K$

1 Brown CH, Berndt D, Brinales JM, et al. Evaluating the evidence of effectiveness for preventive interventions: using a registry system to influence policy through science. Addict Behav 2000;25:955-64.

2 Steiner H. Practice parameters for the assessment and treatment of children and adolescents with conduct disorder. American Academy of Child and Adolescent Psychiatry. J Am Acad Child Adolesc Psychiatry 1997;36:122S-39S. 